Heymans, C., Bouckaert, J. J.: Les chémorécepteurs du sinus carotidien. Ergeb. Physiol. 41, 28 (1939).

Isfort, A., Knoche, H.: Tumoren des Glomus caroticum. Bruns' Beitr. klin. Chir. 212, 417 (1966).

Knoche, H., Alfes, H., Möllmann, H., Reisch, J.: On the biogenic amines in the carotid body: Identification of dopamine by mass spectrometry. Experientia (Basel) 25, 516 (1969).

Le Compte, P. M. : Tumors of the carotid body and related structures (chemoreceptor system). In: Atlas of tumor pathology, Seat IV, Fasc. 16, 40 pp. Subcommittee on Oncology of the Committee of Pathology of the National Research Councill, Armed Forces Institute of Path., Washington 1951.

Linder, F.: Tumoren der Carotisdrüse. Langenbecks Arch. klin. Chir. 276, 156 (1953).

Martorell, F.: Tumor of the carotid body. Angiology 7, 228 (1956).

Masson, P.: Tumeurs humaines. Histologie-diagnostics et techniques. Maloine, Paris 2, 1216 (1956).

Monroe, R. S.: The natural history of carotid body tumors and their diagnosis and treatment. Brit. J. Surg. 37, 445 (1950).

Nakayama, K.: Die Exstirpation des Carotisknotens zur Behandlung des Asthma bronchiale. Chirurg 29, 180 (1958).

Pfarschner, W., Stresemann, E.: Extirpation des Glomus caroticum bei Asthma bronchiale mit längerer Nachbeobachtung. Klin. Wschr. 43, 1223 (1965).

Schnepper, E.: Pers. Mitteilung, 1970.

Schopp, R.: Primäre Geschwülste in der Karotisgabel. Münch. med. Wschr. 30, 1558 (1969).

Sunder-Plassmann, P.: Der Nervenapparat des Sinus caroticum und des Glomus caroticum vom Menschen der verschiedenen Altersstufen und Foetus humanus von verschiedenen Tieren. Verh. dtsch. Ges. Kreisl.-Forsch. 69, 3 (1933).

- Klinik und Neuromorphologie der Glomustumoren. Langenbecks Arch. klin. Chir. 265, 115 (1950).

- Sympathicuschirurgie. Stuttgart: Thieme 1953.

Tiedge, F., Scheiffarth, F., Schmid, E.: Ưber eine Sonderform von hormonal aktiven Tumoren des Glomus jugulare-tympanicum. Z. Laryng. Rhinol. 43, 271 (1964). Ausführliche Literaturübersicht kann bei den Verff. angefordert werden.

Prof. Dr. P. Sunder-Plassmann

Chirurg. Klinik und Poliklinik der Westfälischen Wilhelms-Universität BRD-4400 Münster, Jungeblodtplatz 1 Deutschland

\title{
Erratum
}

\section{Die Druckverhältnisse im unverletzten Hüftgelenk und bei medialen Schenkelhalsfrakturen}

Thre Bedeutung für die präoperative Behandlung

W. J. EwerwaHN und E. G. SuREx, Hamburg

Langenbecks Arch. für Chir., 327, 847-851 (Kongreßbericht 1970)

Durch ein bedauerliches Versehen fehlen die Hinweise auf diese Arbeit im Inhaltsverzeichnis, in der Rednerliste und im Sachregister des Bandes.

Die Leser werden um handschriftlichen Nachtrag gebeten. 